\title{
Análisis de las relaciones de los procesos logísticos de un grupo de micro, pequeñas y medianas empresas manufactureras colombianas
}

\author{
MÁRquEZ GutiÉRREZ, MATEO \\ Universidad EAFIT (Colombia) \\ Correo electrónico: mmarque8@eafit.edu.co \\ CARMONA GONZÁLEZ, GUILLERMO LEÓN \\ Universidad EAFIT (Colombia) \\ Correo electrónico: gcarmona@eafit.edu.co \\ Castro Zuluaga, Carlos Alberto \\ Universidad EAFIT (Colombia) \\ Correo electrónico: ccastro@eafit.edu.co
}

\begin{abstract}
RESUMEN
El presente artículo tiene como propósito explorar la relación que existe entre un grupo de procesos logísticos importantes de micro, pequeñas y medianas empresas (Mipymes) manufactureras colombianas. A través de un número reducido de factores se simplifica la visión de los análisis realizados a este tipo de empresas tanto desde la práctica como desde la literatura. Para ello se utiliza una metodología basada en análisis estadísticos multivariados. El uso de análisis de elementos permite tener una visión general y descriptiva de los procesos logísticos empresariales. El análisis factorial exploratorio, por su parte, permite obtener un conjunto de factores que explican mejor los procesos, mientras un análisis factorial confirmatorio permite corroborar la validez de dichos factores en una muestra de Mipymes.

Los principales hallazgos consideran cuatro factores principales que mejor representan y relacionan los procesos logísticos y de impacto logístico considerados para las Mipymes con un alto nivel de validez estadístico. Esta investigación considera un número limitado de empresas manufactureras y asume que los procesos que se tomaron en cuenta representan la logística de una empresa, de acuerdo con la literatura. Finalmente, se presenta la simplificación, mediante análisis estadísticos, de los problemas logísticos de Mipymes manufactureras en mercados emergentes como la principal aportación del presente artículo.

Palabras clave: análisis factorial, mipymes, macroprocesos logísticos, análisis de elementos, correlación de variables logísticas.
\end{abstract}

Clasificación JEL: C10; M10.

MSC2010: 62P30.

Artículo recibido el 12 de noviembre de 2019 y aceptado el 3 de julio de 2020. 


\title{
Analysis of the relationships of the logistics processes of a group of micro, small and medium colombian manufacturing companies
}

\begin{abstract}
The purpose of this article is to explore the relationship that exists between a group of important logistics processes of Colombian micro, small and mediumsized manufacturing companies (MSMEs). Through a reduced number of factors, the vision of the analyzes carried out on this type of companies is simplified both from practice and from literature. For this, a methodology based on multivariate statistical analysis is used. The use of element analysis allows to have a general and descriptive vision of the business logistics processes. The exploratory factor analysis, for its part, allows obtaining a set of factors that better explain the processes, while a confirmatory factor analysis allows corroborating the validity of these factors in a sample of MSMEs.

The main findings consider four main factors that best represent and relate the logistics and logistics impact processes considered for MSMEs with a high level of statistical validity. This research considers a limited number of manufacturing companies and assumes that the processes that were taken into account represent the logistics of a company, according to the literature. Finally, the simplification, through statistical analysis, of the logistics problems of manufacturing MSMEs in emerging markets is presented as the main contribution of this article.
\end{abstract}

Keywords: factor analysis, MSMEs, logistic macroprocesses, element analysis, logistic variable correlation.

JEL classification: C10; M10.

MSC2010: 62P30.

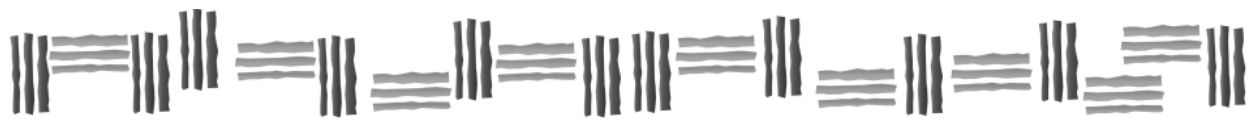




\section{Introducción.}

La logística es una disciplina que se ha considerado frecuentemente como fuente para alcanzar una resiliencia organizacional (Barbosa \& Musetti, 2012). Esta disciplina puede llegar a representar hasta un $20 \%$ de los costos totales de una empresa (Tran, 2019). Este porcentaje en muchos casos puede llegar a ser mayor a causa de la falta de visión de las empresas para solucionar problemas empresariales de raíz y aumenta aún más cuando son empresas de tamaños reducidos (Gelinas \& Bigras, 2004) y en economías no desarrolladas.

Logística a diferencia del concepto de cadena de suministro hace referencia únicamente a la administración de información, materiales, dinero, energía y tiempo al interior de la unidad empresarial. Se considera como un factor importante para el aumento de la competitividad de las empresas, así como por su capacidad de incentivar el desarrollo socioeconómico de los países y sus respectivas regiones (Olivos, Carrasco, Flores, Moreno \& Nava, 2015). Corresponde a un área de interés para todo tipo de empresas, en vista de que se compone de la gestión de elementos necesarios para la existencia y correcto funcionamiento de éstas (Yang \& $\mathrm{Lu}, 2017$ ). El mayor interés nace gracias a su potencial de apalancamiento dado en gran parte por su relevancia en los costos organizacionales (Puertas, Martí \& García, 2014).

Tanto desde el sector público como del privado históricamente han existido cientos de iniciativas, así como trabajos colaborativos que buscan un apalancamiento de la logística para la mejora de la empresa. El creciente interés de las organizaciones por la llamada Industria 4.0 y su influencia sobre la logística han generado que hoy por hoy las iniciativas que se llevan a cabo le dediquen una mayor atención a la logística (Hofmann \& Rüsch, 2017).

Así, existen numerosas aproximaciones de estudio a la logística y a sus áreas de interés desde diferentes modelos, metodologías, estrategias y técnicas. Éstas se basan en el uso de todo tipo de variables que en muchos casos coinciden entre aproximaciones, mientras en otras se genera polémica sobre su relevancia o redundancia de estudio. No existe por su parte una entidad encargada de dictaminar los procesos o variables que deben ser considerados a la hora de estudiar el ámbito logístico en las empresas. Uno de los estudios empíricos más relevantes es el de Croxton, García-Dastugue, Lambert y Rogers (2001) quienes han establecido un fundamento en cuanto a procesos logísticos y en términos generales de cadena de suministro se refiere. Para hacer frente a esto, se plantean metodologías en su mayoría estadísticas como el análisis de varianza (ANOVA) y los análisis factoriales que permiten la simplificación y reducción de variables para justificar su importancia de estudio (Zimon, Madzik \& Sroufe, 2019).

Para la implementación de análisis factorial en términos de cadena de suministro, autores como Valadares de Oliveira, Bronzo Ladeira, y McCormack (2011) desarrollan un modelo de madurez de cadena de suministro, basado en procesos propuestos por el "Supply Chain Council" (hoy APICS), donde a partir de 90 indicadores de capacidad de proceso se hayan 16 factores a través de un análisis factorial exploratorio. Estos 16 factores medidos a través de índices resultan ajustarse bastante bien a una serie de datos recolectados por dicho autor. Finalmente, se validan los factores por medio de una implementación en ambientes reales. Otros enfoques recientes en cadena de suministro incluyen desde la estandarización de los sistemas de administración con el fin de simplificar un proceso tan complejo como éste (Zimon et al., 2019), hasta el uso tanto de análisis factorial exploratorio como confirmatorio para validar factores que benefician la integración de cadenas de suministro de ventas al por menor desde la perspectiva logística (Song, Song \& Sun, 2019).

En términos logísticos como tal, se encuentran aproximaciones como las de Yang y Lu (2017) quienes centran los esfuerzos en resumir 12 competencias que permiten la evolución logística en las organizaciones en tan solo 6 factores. Al igual que otros autores, estos destacan la obtención de factores como un medio para facilitar la toma de decisiones, en especial aquellas complejas o que involucran recursos. Asimismo, se presentan aproximaciones más específicas a nivel logístico que se enfocan en modelos para la planeación estratégica para la implementación de sistemas de información logístico 
(Kitsios, Kamariotou, Madas, Fouskas \& Manthou, 2019). Esta aproximación utiliza encuestas con escala Likert y un análisis factorial confirmatorio como herramientas para simplificar más de 20 variables en cinco factores principales. Igualmente, se ha utilizado análisis factorial para identificar los factores principales de capacidad logística en Micro, Pequeñas y Medianas Empresas (Mipymes) (Granadillo, Acosta \& Mart, 2018). En éste se realiza y valida un análisis factorial exploratorio y se usa el coeficiente alfa de Cronbach para encontrar diez factores sobre los que se sugiere hacer un esfuerzo para mejorar la capacidad logística.

En general, en entornos de pequeñas organizaciones como tal el análisis factorial es una metodología que se utiliza sin discriminación alguna. Algunos casos representativos son el uso de análisis factorial confirmatorio para la identificación de factores relevantes en las características de las pequeñas organizaciones (Jovanović, Arsić \& Nikolić, 2018) y la forma en la que les permite facilitar el proceso de toma de decisiones al interior de la organización agilizando el crecimiento y la internacionalización (Kitsios et al., 2019; Laufs, Bembom \& Schwens, 2016). Laufs et al. (2016) destacan cómo en estos casos los factores responden a la propia lógica que puede presenciarse en las realidades empresariales, obteniendo resultados válidos a partir de ello.

Esta investigación parte de un modelo de evaluación de madurez logística para Mipymes manufactureras. El modelo presenta una recopilación de una gran diversidad de procesos o también llamadas variables, tanto logísticas como de impacto logístico. A partir de ellas se busca determinar un número estadísticamente menor de nuevas variables o también factores, a través tanto de análisis factorial exploratorio y confirmatorio, como otros análisis, que representen y resuman las variables consideradas originalmente.

El artículo se organiza de la siguiente manera. En la Sección 2 se presentan los métodos utilizados en el estudio estadístico y explica cómo fue el proceso para recolectar los datos. La Sección 3 muestra los resultados obtenidos de los diferentes análisis realizados, principalmente los análisis factoriales y las validaciones de estos. Por su parte, la Sección 4 presenta las discusiones generadas a partir de los resultados obtenidos, contrastando, comparando y procurando explicar dichos resultados. Finalmente, las conclusiones, aprendizajes y futuros estudios del caso se presentan en la Sección 5 del presente artículo.

\section{Métodos.}

Los métodos utilizados en la investigación van tanto desde las técnicas de recolección de datos como su posterior orden y análisis estadístico.

\subsection{Recolección de datos y características de las empresas.}

Los datos se obtienen a partir de una herramienta de autoevaluación de procesos logísticos de Mipymes de la ciudad de Medellín, Colombia. Esta herramienta de autoevaluación hace parte de un modelo de evaluación de madurez de procesos logísticos que consta de un total de 48 procesos entre ellos logísticos y no logísticos propiamente pero que afectan el desempeño logístico de la empresa. Este modelo de evaluación se encuentra fundamentado en estudios previos al respecto (Battista \& Schiraldi, 2013; Croxton et al., 2001).

Las empresas se registran a través de una plataforma diseñada para la evaluación de sus procesos. Éstas diligencian un formulario electrónico (Valadares de Oliveira et al., 2011) en el que califican los 48 procesos propuestos en una escala de madurez que va entre cero y cinco, donde cero hace referencia a cuando los procesos son caóticos y cinco cuando estos se encuentran en optimización o en un proceso de mejora constante (Battista \& Schiraldi, 2013; Kitsios et al., 2019). Como medio para incentivar la participación de las empresas y una evaluación que fuera transparente, éstas recibían retroalimentaciones como apoyo en la mejora de sus procesos y una comparación anónima tipo 
benchmarking con otras organizaciones. El acceso a la plataforma era voluntario, gratuito y sin ningún tipo de restricción; posteriormente las empresas y los datos son filtrados y limpiados para los respectivos análisis, asegurando la calidad de éstos.

Empresas de servicio, consultoras, extracción de bienes primarios, financieras, gubernamentales y en general grandes empresas son excluidas de la investigación. Se consideran únicamente Mipymes manufactureras por su impacto en las economías locales (Nyoni \& Bonga, 2018) y por ser el foco de interés de la investigación. Adicionalmente, se verifica que las empresas seleccionadas cuenten con las herramientas necesarias para llevar a cabo una adecuada evaluación de sus procesos, así como que no cuenten con problemas a nivel financiero, legal o político que pueda interferir con la evaluación.

La selección de las empresas se hace a modo de muestreo no probabilístico de tipo por conveniencia y se realiza a través de contactos personales y profesionales, y por difusión de ésta. El método usado con las encuestas para la autoevaluación a través de formularios corresponde a una de las practicas más adecuadas a la hora de evaluar unidades de negocio y empresas (Sledgianowski, Luftman \& Reilly, 2011; Yang \& Lu, 2017). Este método de evaluación elimina presiones por la presencia de terceros e impresiones falsas del negocio percibidas durante el desarrollo de la evaluación que se realiza presencialmente (Cronemyr \& Danielsson, 2015). Posteriormente, se realiza una validación presencial al $9 \%$ de las empresas sobre los datos recolectados para confrontar las evaluaciones realizadas por cada una de ellas.

\subsection{Métodos estadísticos implementados.}

Entre los métodos estadísticos utilizados se encuentra el análisis de elementos que busca describir superficialmente la naturaleza las variables y las relaciones entre ellas a partir de las observaciones consideradas. De igual importancia se hace uso del análisis factorial que determina los factores que mejor representan las variables. Todos los análisis se plantean para realizarse sobre una muestra de 55 empresas, en donde cada una calificó 48 procesos en total.

\subsubsection{Análisis de elementos}

El primer análisis realizado parte del uso del concepto de correlación. Éste se utiliza para evaluar la fuerza y dirección de cambio entre dos variables cuantitativas. Un valor alto y positivo de correlación indica una relación directamente proporcional, mientras un valor alto y negativo indica una relación inversamente proporcional. Un valor cercano a cero evidencia que las variables no tienen ninguna relación entre ellas y que parecen verse afectadas por eventos totalmente diferentes. Una medida de correlación de variables con valor absoluto por encima de 0,7 refleja que éstas se encuentran estrechamente ligadas y que, probablemente se ven afectadas por los mismos fenómenos (Comrey \& Lee, 2013). Igualmente, medidas de correlación superiores a 0,5 muestran una relación medianamente fuerte entre las variables. Tanto las medidas de correlación de Pearson, Spearman, como otras, se utilizan para medir esta relación entre variables y construir matrices de correlación que permitan un panorama detallado del nivel como diferentes variables se ven afectadas en igual medida por otras.

Por otra parte, coeficientes como el alfa de Cronbach, el omega de McDonald, el lambda de Guttman, el beta de Revelle, entre otros, se utilizan como medios para evaluar la consistencia, uniformidad y confianza de una herramienta de medición utilizada (Revelle \& Zinbarg, 2009). En especial estos dos primeros coeficientes, cuanto mayor sean, mejor será la capacidad en cuanto a precisión y pertinencia de la herramienta utilizada para medir las respectivas variables, donde se esperan valores cercanos o superiores a 0,7 (Jovanović et al., 2018). Especialmente el coeficiente de alfa de Cronbach debe ser lo más cercano a 0,95 como sea posible. Sin embargo, un coeficiente menor no significa una baja calidad del estudio ni de la herramienta utilizada (Pennypacker \& Grant, 2003).

Es posible, además, probar diferentes escenarios donde al omitir alguna variable se mejora considerablemente el valor del coeficiente de alfa de Cronbach. La variable que al ser omitida mejora el coeficiente demuestra, a través de las mediciones obtenidas, afectar los datos. Coeficientes como el 
omega de McDonald son análogos al alfa de Cronbach, basando su medida en las cargas de factores a extraer de los datos, siendo en algunos casos más pertinente su uso al no sesgarse por las varianzas de las variables, como sucede en el coeficiente alfa (Revelle \& Zinbarg, 2009).

\subsubsection{Análisis factorial}

A diferencia del análisis de componentes principales (PCA) que pretende explicar la mayor parte de la varianza total como sea posible de un grupo de variables, el análisis factorial define las variables originales como combinaciones lineales de factores que explican las correlaciones entre las variables.

Los factores son calculables y ejercen un nivel de influencia sobre las variables expresado a través de coeficientes que indican el grado de representación de la variable en el factor. La varianza total de la medición de las variables que los factores deben explicar debe ser lo suficiente para representar fielmente las variables originales, pero que a su vez las describa en un número pequeño y limitado de factores.

Para asegurar un correcto desarrollo de un análisis factorial se sugiere que el número de observaciones sea mayor al número de variables. Autores tales como Lloret-Segura, Ferreres-Traver, Hernández-Baeza y Tomás-Marco (2014) sugieren tener una relación de observaciones a variables de 5 a 1.

El número de factores a extraer del análisis factorial exploratorio se determina en este caso con aquellos que logren explicar lo más cercano al $80 \%$ de la varianza total de la medición de las variables como sea posible. Igualmente, apoyado en el criterio de Kaiser para determinar el número de factores, que establece la elección de factores con valores propios mayores a uno (Williams, Onsman \& Brown, 2010).

\section{Resultados.}

Los resultados obtenidos constan inicialmente de una simplificación de los procesos en macroprocesos. Las estadísticas descriptivas de los macroprocesos se presentan luego de la simplificación. Posteriormente, a partir de la estadística se determinan los factores que mejor representan los macroprocesos y en consecuencia los procesos que los componen, para la posterior validación de estos factores.

Por otro lado, respecto a las encuestas realizadas, el $60 \%$ eran microempresas, el 32,73\% pequeñas empresas y un 7,27\% medianas empresas. Las personas que respondían la encuesta eran gerentes o personas con conocimientos completos de las empresas; de estos el $56 \%$ eran hombres con un promedio de edad de 41,89 años y el 44\% mujeres con un promedio de edad de 46,82 años.

\subsection{Ponderación de procesos y cálculo de desempeño macroprocesos.}

La presencia de gran cantidad de procesos dificulta cualquier tipo de análisis estadístico que se realice. El modelo base utilizado para evaluar Mipymes consta de 48 procesos agrupados en 10 macroprocesos. Los procesos de cada macroproceso reciben una ponderación. Esta ponderación corresponde al inverso de la proporción de la varianza de cada uno de los procesos respecto al total de procesos de un macroproceso (1), para la muestra de 55 Mipymes. La ponderación dada permite dar un mayor peso a los procesos con una menor varianza en cada macroproceso, de manera que se tenga consistencia entre los macroprocesos de diferentes empresas.

$$
W_{i}=\frac{1}{S_{i}^{2} \cdot \sum \frac{1}{s_{i}^{2}}}
$$


Cada macroproceso tiene entre cuatro y ocho procesos. El cálculo de la madurez de los macroprocesos corresponde a la suma ponderada de sus procesos. Este cálculo se realiza con el fin de representar cada macroproceso de manera integral a partir de un grupo de procesos y estar en la capacidad de llevar a cabo un análisis factorial con un número considerablemente menor de variables.

\subsection{Estadísticas generales de los procesos.}

La media, la desviación estándar, tamaño de la muestra y demás estadísticas descriptivas de cada uno de los macroprocesos se presentan en la Tabla 1. Los estadísticos descriptivos permiten contar con un acercamiento superficial inicial del comportamiento de los macroprocesos en la muestra de Mipymes.

Tabla 1. Estadísticas descriptivas de los macroprocesos.

\begin{tabular}{|l|c|c|c|c|c|c|c|}
\hline Variable & Media & Desviación Estándar & Mediana & Mínimo & Máximo & $25 \%$ & $75 \%$ \\
\hline Almacenes & 2,38 & 0,77 & 2,40 & 1,15 & 3,88 & 1,65 & 2,87 \\
\hline Inventarios & 2,22 & 0,66 & 2,32 & 0,84 & 3,63 & 1,72 & 2,65 \\
\hline Abastecimiento & 2,15 & 0,66 & 2,26 & 0,76 & 3,49 & 1,68 & 2,64 \\
\hline Servicio al cliente & 2,03 & 0,75 & 2,02 & 0,63 & 4,12 & 1,50 & 2,47 \\
\hline Planeación & 2,02 & 0,84 & 1,89 & 0,31 & 3,51 & 1,38 & 2,76 \\
\hline RRHH & 2,50 & 0,60 & 2,40 & 1,40 & 4,10 & 2,11 & 2,85 \\
\hline Ventas & 1,46 & 0,75 & 1,32 & 0,44 & 3,56 & 0,88 & 1,88 \\
\hline Producción & 2,39 & 0,43 & 2,43 & 1,59 & 3,64 & 2,10 & 2,66 \\
\hline Organizacional & 2,10 & 0,52 & 2,12 & 0,94 & 3,30 & 1,83 & 2,40 \\
\hline Externas & 1,50 & 0,66 & 1,38 & 0,34 & 3,10 & 1,04 & 2,10 \\
\hline
\end{tabular}

Fuente: Elaboración propia.

Los valores correspondientes a cada uno de los estadísticos descriptivos presentados en la Tabla 1 no se encuentran representados en ninguna unidad de medida. Los valores de los estadísticos responden a la escala adimensional creada para evaluar cada los procesos. Asimismo, todos los análisis siguientes realizados sobre los datos de los macroprocesos presentan esta característica de adimensionalidad. La Tabla 1 permite extraer conclusiones iniciales generales acerca de los macroprocesos. Con estos datos es posible determinar, de la muestra de 55 Mipymes, en términos generales cuál es el macroproceso más débil y cuál es el más fuerte, ya sea utilizando los cuartiles o la media como puntos de corte. Asimismo, es posible determinar el macroproceso que es más estándar entre las organizaciones con ayuda de la desviación estándar.

\subsection{Correlación y covarianza de variables.}

Después de comprender el comportamiento individual de los macroprocesos, se analizan las relaciones entre ellos. La Tabla 2 muestra las correlaciones que existen entre los diferentes macroprocesos. El concepto de correlación se entiende como la medida en la que se relacionan dos variables o macroprocesos en este caso. Las correlaciones de un macroproceso consigo mismo se omiten, al igual que la mitad de la matriz por la redundancia que significa. 
Tabla 2. Matriz de correlaciones de macroprocesos.

\begin{tabular}{|l|c|c|c|c|c|c|c|c|c|}
\hline & Almacenes & Inventarios & Abastecimiento & $\begin{array}{c}\text { Servicio al } \\
\text { cliente }\end{array}$ & Planeación & RRHH & Ventas & Producción & Organizacional \\
\hline Inventarios & 0,69 & & & & & & & & \\
\hline Abastecimiento & 0,50 & 0,47 & & & & & & & \\
\hline $\begin{array}{l}\text { Servicio al } \\
\text { cliente }\end{array}$ & 0,07 & $-0,02$ & 0,24 & & & & & & \\
\hline Planeación & 0,65 & 0,64 & 0,63 & 0,16 & & & & & \\
\hline RRHH & 0,45 & 0,27 & 0,26 & 0,20 & 0,33 & & & & \\
\hline Ventas & 0,32 & 0,40 & 0,36 & 0,23 & 0,68 & 0,31 & & & \\
\hline Producción & 0,43 & 0,39 & 0,35 & 0,10 & 0,37 & 0,38 & 0,22 & & \\
\hline Organizacional & 0,43 & 0,36 & 0,41 & 0,32 & 0,48 & 0,55 & 0,58 & 0,47 & \\
\hline Externas & 0,50 & 0,47 & 0,53 & 0,09 & 0,60 & 0,22 & 0,41 & 0,28 & 0,43 \\
\hline
\end{tabular}

Fuente: Elaboración propia.

Los valores de correlación en la Tabla 2 se expresan a través de correlaciones de Pearson. Las correlaciones de Pearson se denotan a través de coeficientes que pueden ser tanto valores positivos como negativos, que indican una relación inversamente proporcional entre macroprocesos para este caso. Estos valores representan coeficientes que indican el cambio de dos macroprocesos por un mismo suceso o fenómeno. Para esta matriz de correlaciones, se cuenta con un determinante de 0,009 , lo suficientemente bajo como para justificar la posibilidad de realizar un análisis factorial. Del mismo modo, el determinante de la matriz de correlaciones de Spearman que no requiere el supuesto de normalidad de los datos es igual a 0,007 , reforzando lo mencionado anteriormente con respecto a la posibilidad de realizar análisis factorial.

Por su parte, tanto las correlaciones de Pearson (Tabla 2) como las correlaciones de Spearman que presentan un valor de correlación superior a 0,5 son estadísticamente significativas con un valor $p$ de 0,05 . Por otra parte, el $62,86 \%$ de las correlaciones de Pearson inferiores a 0,5 son significativas, en comparación con un 70,59\% de las correlaciones de Spearman. Se evidencia que los macroprocesos logísticos seleccionados cuentan con fuertes relaciones entre sí, respaldadas en los datos de la muestra.

De forma similar la Tabla 3 presenta las covarianzas de los macroprocesos a través de una matriz. Esta matriz funciona como medio adicional para afirmar que los datos con los que se cuenta de la muestra de empresas son aptos para realizarles análisis de factores.

Tabla 3. Matriz de covarianza de macroprocesos.

\begin{tabular}{|c|c|c|c|c|c|c|c|c|c|c|}
\hline & Almacenes & Inventarios & Abastecimiento & $\begin{array}{c}\text { Servicio al } \\
\text { cliente }\end{array}$ & Planeación & RRHH & Ventas & Producción & Organizacional & Externas \\
\hline Almacenes & 0,60 & & & & & & & & & \\
\hline Inventarios & 0,35 & 0,43 & & & & & & & & \\
\hline Abastecimiento & 0,26 & 0,20 & 0,44 & & & & & & & \\
\hline $\begin{array}{l}\text { Servicio al } \\
\text { cliente }\end{array}$ & 0,04 & $-0,01$ & 0,12 & 0,56 & & & & & & \\
\hline Planeación & 0,42 & 0,35 & 0,35 & 0,10 & 0,71 & & & & & \\
\hline RRHH & 0,21 & 0,11 & 0,10 & 0,09 & 0,17 & 0,35 & & & & \\
\hline Ventas & 0,19 & 0,20 & 0,18 & 0,13 & 0,43 & 0,14 & 0,56 & & & \\
\hline Producción & 0,14 & 0,11 & 0,10 & 0,03 & 0,13 & 0,10 & 0,07 & 0,18 & & \\
\hline Organizacional & 0,17 & 0,12 & 0,14 & 0,13 & 0,21 & 0,17 & 0,23 & 0,11 & 0,27 & \\
\hline Externas & 0,25 & 0,21 & 0,23 & 0,04 & 0,34 & 0,09 & 0,20 & 0,08 & 0,15 & 0,44 \\
\hline
\end{tabular}

Fuente: Elaboración propia. 
A diferencia de los valores presentados en la Tabla 2, los datos de la matriz de covarianza no se encuentran estandarizados, por lo que es difícil determinar cuándo es fuerte la covarianza entre macroprocesos. Sin embargo, los valores suficientemente pequeños en la matriz de covarianza de macroprocesos se interpretan como si fueran cero, de forma que sea posible la realización de otros análisis estadísticos. Nuevamente, que entre dos variables o macroprocesos se tenga una medida baja de covarianza representa una semejanza en el comportamiento de éstos, y contrastado con lo evidenciado en la Tabla 2 hacen referencia a hechos similares.

\subsection{Consistencia y omisión de variables.}

Adicional a la caracterización individual de macroprocesos y sus relaciones, el conjunto de datos recolectados presenta un alfa de Cronbach de 0,86 y un omega de McDonald de 0,91, reflejando una alta consistencia de la herramienta utilizada a través de ambos coeficientes. Basados únicamente en el alfa de Cronbach es posible, con el fin de mejorar la consistencia del estudio, omitir ciertos macroprocesos. La Tabla 4 muestra todos los posibles escenarios donde al omitir algún macroproceso el alfa de Cronbach puede mejorar o empeorar. Idealmente se busca no recurrir a omitir macroprocesos, siempre y cuando el impacto en la consistencia que se puede adquirir al omitir variables no sea significativo.

Tabla 4. Estadísticas de variables omitidas.

\begin{tabular}{|l|c|c|c|c|c|}
\hline Variable omitida & Media total & $\begin{array}{c}\text { Desviación } \\
\text { estándar total }\end{array}$ & $\begin{array}{c}\text { Correlación } \\
\text { total por } \\
\text { elemento }\end{array}$ & $\begin{array}{c}\text { Correlación } \\
\text { múltiple } \\
\text { cuadrada }\end{array}$ & $\begin{array}{c}\text { Alfa de } \\
\text { Cronbach }\end{array}$ \\
\hline Almacenes & 18,36 & 3,89 & 0,67 & 0,63 & 0,83 \\
\hline Inventarios & 18,52 & 4,01 & 0,62 & 0,56 & 0,84 \\
\hline Abastecimiento & 18,59 & 4,00 & 0,63 & 0,48 & 0,84 \\
\hline Servicio al cliente & 18,72 & 4,23 & 0,21 & 0,18 & 0,88 \\
\hline Planeación & 18,73 & 3,76 & 0,79 & 0,75 & 0,82 \\
\hline RRHH & 18,24 & 4,14 & 0,47 & 0,38 & 0,85 \\
\hline Ventas & 19,28 & 3,97 & 0,59 & 0,63 & 0,84 \\
\hline Producción & 18,36 & 4,23 & 0,48 & 0,33 & 0,85 \\
\hline Organizacional & 18,64 & 4,08 & 0,67 & 0,60 & 0,84 \\
\hline Externas & 19,24 & 4,03 & 0,59 & 0,44 & 0,84 \\
\hline
\end{tabular}

Fuente: Elaboración propia.

Entre las medidas presentadas en cada uno de los escenarios donde se omiten variables o macroprocesos se encuentran la media total, desviación estándar total y las correlaciones totales por elemento y múltiple cuadrada. Estas medidas permiten determinar si justifica, con el propósito de mejorar el análisis, dejar de considerar ciertos macroprocesos. El principal criterio para determinar la omisión de variables en este caso es la consistencia adquirida en el alfa de Cronbach. Un segundo criterio en caso de ser necesario es el aumento de la madurez de los macroprocesos percibida por medio de la media total. Solamente hay un escenario en el que, al omitir el respectivo macroproceso se mejoran las consistencias de las mediciones de madurez realizadas; es el caso del macroproceso de Servicio al Cliente. El potencial de mejora de la herramienta de medición, sin involucrar la aptitud de los datos para realizar análisis factorial, es de un alfa 0,88 respecto a un 0,86 original. 


\subsection{Cargas de factores $y$ análisis factorial.}

Finalmente, no se justifica omitir ningún macroproceso, y en conjunto con la evidencia presentada con anterioridad, se permite apuntar a realizar un análisis factorial. Adicionalmente, pruebas realizadas a los datos como la prueba de esfericidad de Bartlett con un valor $\mathrm{p}$ de $2.54 \times 10^{-23}$ y la prueba de KaiserMeyer-Olkin (KMO) con una adecuación muestral de 0,82 permiten reforzar la posibilidad de realizar un análisis factorial exploratorio (Yang \& Lu, 2017). La Tabla 5 presenta las cargas o influencias de cada uno de los factores, su varianza o valor propio y el porcentaje de la varianza total que cada factor explica. Igualmente se presentan las comunalidades de cada uno de los macroprocesos; éstas representan la varianza explicada de cada uno de los macroprocesos en los factores definidos. La suma de los porcentajes de varianza de los factores representa el porcentaje acumulado de la varianza total de los macroprocesos que los factores logran explicar.

Tabla 5. Cargas de factores no rotados y comunalidades.

\begin{tabular}{|c|c|c|c|c|c|}
\hline Variable & FACTOR1 & FACTOR2 & FACTOR3 & FACTOR4 & Comunalidad \\
\hline Almacenes & 0,32 & 0,79 & $-0,08$ & 0,23 & 0,78 \\
\hline Inventarios & 0,40 & 0,63 & $-0,22$ & 0,19 & 0,64 \\
\hline Abastecimiento & 0,36 & 0,61 & $-0,06$ & $-0,41$ & 0,66 \\
\hline $\begin{array}{l}\text { Servicio al } \\
\text { cliente }\end{array}$ & 0,23 & 0,07 & 0,29 & $-0,28$ & 0,22 \\
\hline Planeación & 0,68 & 0,54 & $-0,23$ & $-0,10$ & 0,82 \\
\hline RRHH & 0,31 & 0,40 & 0,42 & 0,17 & 0,46 \\
\hline Ventas & 1,00 & 0,00 & 0,00 & 0,00 & 1,00 \\
\hline Producción & 0,22 & 0,50 & 0,26 & 0,05 & 0,37 \\
\hline Organizacional & 0,58 & 0,38 & 0,55 & $-0,03$ & 0,79 \\
\hline Externas & 0,41 & 0,51 & $-0,08$ & $-0,18$ & 0,47 \\
\hline Varianza & 2,56 & 2,50 & 0,75 & 0,40 & 6,21 \\
\hline$\%$ Var & 0,26 & 0,25 & 0,08 & 0,04 & 0,62 \\
\hline
\end{tabular}

Fuente: Elaboración propia.

Las cargas de factores no rotados es el primer resultado del análisis factorial exploratorio realizado a los datos. Allí se presentan las llamadas influencias que realizan cada uno de los factores sobre los diferentes macroprocesos. Para la extracción de los factores se utilizó el método de máxima verosimilitud, que proporciona las estimaciones de los parámetros que con mayor probabilidad ha producido la matriz de correlaciones (Kitsios et al., 2019). El método de máxima verosimilitud requiere una distribución normal multivariada de los datos. A través de los métodos de Doornik-Hansen, Anderson-Darling y Cramer-von Mises usados como pruebas de normalidad multivariada, se muestra que el conjunto de datos presenta normalidad multivariada con un valor $p$ de significancia de 0,05 . Los factores seleccionados comprenden de esta manera más del $60 \%$ de los macroprocesos, al mismo tiempo que se conserva un número significativamente menor de factores respecto a las variables.

El método de rotación Equamax permite maximizar el aprovechamiento del potencial de los factores. Éste minimiza tanto el número de variables que saturan alto un factor como el número de factores necesarios para explicar una variable (Osborne, Costello \& Kellow, 2011). El resultado de los factores rotados se muestra en la Tabla 6, que cuenta con una estructura de los datos similar al de la Tabla 5. El método de rotación ortogonal seleccionado corresponde a una variante de la rotación Varimax utilizada tradicionalmente en la literatura (Keh, Nguyen \& Ng, 2007; Yang \& Lu, 2017). 
Los valores presentados en la Tabla 6 corresponden a las cargas de los factores rotados bajo el método ortogonal Equamax. Los factores se rotan a fin de mejorar sus propiedades descriptivas y prescriptivas. Las variaciones y comunalidades de los diferentes factores se muestran al igual que en el método sin rotación. El criterio de Kaiser se utiliza para la selección de la cantidad final de factores, donde se evidencia que los cuatro factores que se extraen presentan un valor propio superior a la unidad y que explican más del 60\% de la varianza (Lloret-Segura et al., 2014). Adicionalmente se evidencia cómo cada macroproceso tiene mayores cargas en algunos factores, haciendo que sea posible simplificar la visión de los macroprocesos considerados inicialmente en un esperado número inferior de factores.

Tabla 6. Cargas de factores con rotación Equamax.

\begin{tabular}{|c|c|c|c|c|c|}
\hline Variable & FACTOR1 & FACTOR2 & FACTOR3 & FACTOR4 & Comunalidad \\
\hline Almacenes & 0,76 & 0,13 & 0,33 & $-0,27$ & 0,78 \\
\hline Inventarios & 0,70 & 0,26 & 0,16 & $-0,24$ & 0,64 \\
\hline Abastecimiento & 0,31 & 0,18 & 0,17 & $-0,71$ & 0,66 \\
\hline Servicio al cliente & $-0,19$ & 0,16 & 0,28 & $-0,29$ & 0,22 \\
\hline Planeación & 0,53 & 0,54 & 0,12 & $-0,48$ & 0,82 \\
\hline RRHH & 0,23 & 0,15 & 0,62 & $-0,08$ & 0,46 \\
\hline Ventas & 0,12 & 0,96 & 0,20 & $-0,16$ & 1,00 \\
\hline Producción & 0,30 & 0,06 & 0,47 & $-0,23$ & 0,37 \\
\hline Organizacional & 0,09 & 0,40 & 0,74 & $-0,27$ & 0,79 \\
\hline Externas & 0,37 & 0,26 & 0,17 & $-0,48$ & 0,47 \\
\hline Varianza & 1,79 & 1,61 & 1,47 & 1,34 & 6,21 \\
\hline$\%$ Var & 0,18 & 0,16 & 0,15 & 0,13 & 0,62 \\
\hline
\end{tabular}

Del mismo modo, se resalta que cada factor ejerce influencia sobre todos los macroprocesos; no obstante, ejercen mayor influencia sobre un grupo de macroprocesos específico. En la práctica, estos factores describen unas circunstancias que afectan en paralelo el desempeño de madurez de los macroprocesos. Al no representar el $100 \%$ de la varianza de los macroprocesos, producto de tener menos factores que macroprocesos, algunos de los factores no generan suficiente influencia sobre ciertos macroprocesos.

La Tabla 7 presenta los resultados de un análisis factorial confirmatorio realizado al modelo obtenido por el análisis factorial exploratorio. Se considera en primera instancia el modelo de cuatro factores y se compara con un modelo de un solo factor para evaluar la pertinencia de estos factores.

Tabla 7. Índices de bondad de ajuste para el modelo de cuatro factores $\left(\mathrm{N}^{\circ}\right.$. 1) y un modelo de un factor (No. 2)

\begin{tabular}{|c|c|c|c|c|c|c|c|c|c|}
\hline Modelo & $\chi^{2}$ & GL & Valor-p & RMSEA & SRMR & CFI & TLI & AIC & BIC \\
\hline 1 & 24,088 & 21 & 0,289 & 0,054 & 0,063 & 0,984 & 0,972 & 715,52 & 761,409 \\
\hline 2 & 51,581 & 27 & 0,003 & 0,135 & 0,082 & 0,87 & 0,827 & 731,013 & 765,429 \\
\hline
\end{tabular}

A través de los índices de bondad de ajuste mostrados en la Tabla 7 es posible corroborar la conformación de los factores generados a través del análisis exploratorio, verificando además la correcta disposición de los macroprocesos. 


\section{Discusión.}

El estudio procede primero con un análisis descriptivo de los macroprocesos logísticos considerados. Luego estudia las relaciones que se evidencian entre los macroprocesos de las diferentes empresas que accedieron a participar del estudio. Finalmente se llega a un análisis de factores que indiquen las dimensiones o factores en las que los macroprocesos pueden ubicarse para facilitar sus futuros estudios o análisis (Gorman \& Primavera, 1983).

El modelo de madurez utilizado para evaluar el desempeño logístico de las empresas consta de 48 procesos en total. Para efectos de los análisis estadísticos realizados, la cantidad de variables dificulta el estudio en vista de que se requieren al menos cinco veces más observaciones para asegurar validez de los mismos (Lloret-Segura et al., 2014). Es por ello por lo que se busca una estrategia para no solo agrupar sino representar numéricamente los 48 procesos en los respectivos 10 macroprocesos con los que se trabajan. Esta forma de representar permite personificar fielmente la totalidad de procesos del modelo. Disponer de 10 variables en lugar de 48, facilita y mejora el manejo de los datos, los análisis y los resultados.

En términos generales el macroproceso que por presentar una media de madurez más baja y puede ser considerado como el más débil en la muestra de empresas analizadas son las ventas. Al evaluarlas dentro de las empresas se consideran las estrategias utilizadas, los niveles de venta respecto a los esfuerzos realizados y los niveles de exportación. Por el contrario, el macroproceso que en general presenta en promedio un mayor desarrollo es el manejo del recurso humano (RRHH). La gestión y manejo del recurso humano considera aspectos como el cuidado, desarrollo, capacidades y niveles de estudio del personal, así como la adecuación de los lugares de trabajo de éste. La causa de que el macroproceso de ventas sea el menos maduro o más débil puede deberse a que son empresas en mercados sumamente competitivos donde las estrategias utilizadas para vender requieren, en el futuro cercano, una revisión cuidadosa, así como las asistencias necesarias para el aumento de las exportaciones. Por el contrario, el macroproceso que evidencia mayor fortaleza se debe a un tema cultural en una región donde las relaciones interpersonales y profesionales suelen ser muy fuertes. Los estudios de procesos comúnmente señalan procesos fuertes y débiles en casos de estudio determinados (Tracey, 1998; Tran, 2019; Wynn, Turner \& Lau, 2013), a diferencia del presente artículo.

La matriz de correlaciones muestra relaciones primarias de alto interés entre los macroprocesos de las Mipymes que en cierta medida apelan a la lógica (Laufs et al., 2016), contando adicionalmente con una significancia estadística. La más fuerte de las relaciones se encuentra entre el manejo de los inventarios y la gestión del almacenamiento como estructura física. Existe evidencia en parte de las empresas validadas presencialmente, que una buena organización del almacenamiento viene acompañada de un mejor manejo de los inventarios. Por lo tanto, en varios casos de la práctica, los macroprocesos de almacenamiento e inventarios se trabajan como una sola área.

La matriz de covarianzas con valores cercanos a cero (Dziuban \& Shirkey, 1974), los cálculos de determinantes de las matrices de correlaciones y pruebas como la KMO y la esfericidad de Bartlett (Yang \& Lu, 2017) son las principales fuentes que permiten indicar que los datos recolectados son aptos para realizar análisis factorial exploratorio y posteriormente confirmatorio. Todos los resultados de estas estrategias aportaron positivamente a este análisis.

Huang y Han (2006) sugieren contar con datos que muestren un alfa de Cronbach superior 0,7 para asegurar una alta consistencia. El modelo de madurez utilizado con los macroprocesos seleccionados presenta una alta consistencia con medidas de alfa de Cronbach de 0,86 y omega de McDonald de 0,91. A pesar de revisar escenarios donde es posible mejorar la consistencia del modelo, basado en el alfa de Cronbach, y por ende la interpretación dada a los macroprocesos, solo se mejora en uno de los escenarios con un alfa $2,3 \%$ mayor. No se justifica por su bajo impacto sobre la consistencia, omitir el macroproceso de servicio al cliente del modelo y del análisis posterior. Ambos coeficientes tienen como resultado valores altos que validan la herramienta, la metodología y los análisis. 
Estudios factoriales específicamente en modelos de madurez en empresa ya se han realizado con anterioridad. El caso de Sledgianowski et al. (2011) donde un análisis factorial sobre un modelo de madurez para evaluar TI en ocho empresas encuentra seis factores claves o el de Valadares de Oliveira et al. (2011) quienes reducen 90 procesos en 16 factores. La selección del número de factores corresponde a lo propuesto por la literatura. Lloret-Segura et al., (2014) sugieren dentro de las técnicas tradicionales comúnmente aceptadas utilizar tanto el criterio de Kaiser como el criterio de la varianza total explicada. Estos métodos para determinar el número de factores resultaron apropiados puesto que permitieron encontrar un número significativamente menor de factores que se ajustaron bastante bien a los datos y a las variables.

El resultado del análisis factorial exploratorio permite determinar y el análisis factorial confirmatorio reconocer un FACTOR1 compuesto por los macroprocesos de Almacenes $(|0,79|)$ e Inventarios $(|0,70|)$, que describe todo lo involucrado con el manejo de materias primas, insumos y producto en proceso dentro de la empresa. El FACTOR2 lo componen Planeación $(|0,54|)$ y Ventas $(|0,96|)$ al describir el desarrollo de las estrategias gerenciales de las empresas que impactan entre otras cosas las ventas y la internacionalización. El FACTOR3 por su parte está compuesto por la gestión del Recurso Humano $(|0,62|)$, los macroprocesos Organizacionales $(|0,74|)$ y Producción $(|0,47| \approx 0,5)$ que describen la cultura, el desarrollo y el papel del personal en la generación de valor tangible de la compañía. Finalmente, el FACTOR4 lo componen el Abastecimiento $(|-0,71|)$ y variables Externas (|$0,48 \mid \sim 0,5)$ que representan las influencias del mercado, la economía y las políticas en el desempeño de la empresa. Los macroprocesos que forman cada uno de los factores que cuentan con rotación Equamax, disponen de cargas iguales o superiores a 0,5 . El único macroproceso que no se ubica dentro de ninguno de los factores por su baja influencia en los factores definidos es el Servicio al Cliente. De forma similar, los macroprocesos originales tienen comunalidades en un gran rango de valores. Sin embargo, esto no representa una preocupación pues a grandes rasgos los macroprocesos presentan altas influencias de los factores establecidos, y sabiendo que cada macroproceso cuenta con una comunalidad superior a 0,2 lo que los hace relevantes para la extracción de factores (Zimon et al., 2019).

Por su parte el análisis factorial confirmatorio permite observar cómo índices de bondad de ajuste como el RMSEA, SRMR, CFI y el TLI en términos generales validan el modelo de cuatro factores obtenido a través del análisis factorial exploratorio (Song et al., 2019). De esta manera se valida tanto la cantidad de factores definidos, como la composición de cada uno de ellos y cómo se ajusta el modelo a los datos recolectados.

\section{Conclusiones.}

La investigación corresponde a un estudio de correlaciones y factores, por lo que no se menciona causalidad de una variable respecto a otra. Todos los valores presentados de los macroprocesos corresponden a un nivel de madurez en una escala entre 0 y 5 . Esta escala permitió y facilitó un análisis y evaluación objetiva de los procesos y macroprocesos empresariales. El cálculo del desempeño de los macroprocesos mediante los procesos que los componen permite reducir la complejidad del problema mientras se visualiza de forma integral y con criterio suficiente a las empresas. Los macroprocesos cuentan con desviaciones estándar bajas, por lo que el promedio resulta ser una medida válida para concluir sobre macroprocesos débiles y fuertes. Tanto el macroproceso más fuerte (Recurso Humano) como el más débil (Ventas) se ven influenciados por las características del ambiente sociocultural y competitivo propio de la muestra de empresas.

La matriz de correlaciones presenta múltiples correlaciones fuertes entre los macroprocesos. A pesar de que los factores explican un conjunto de macroprocesos relacionados, la matriz de correlaciones permite ver relaciones una a una. La matriz de covarianzas respecto a la matriz de correlaciones no muestra información adicional; sin embargo, sirve como apoyo para demostrar relaciones entre variables, entre otros usos. El constructo de madurez se valida con los altos valores del alfa de Cronbach y de la omega de McDonald obtenidos. A pesar de que existe la posibilidad de omitir 
variables para mejorar la consistencia de los macroprocesos y del modelo usado como base, el potencial de mejora que se obtiene no justifica la omisión de variables. Asimismo, el omega de McDonald que se basa en las cargas factoriales de las variables, es lo suficientemente alto como para tener la necesidad de omitir variables.

Los factores resultantes representan las áreas más grandes en las que las Mipymes deben enfocarse para evitar caer en un enfoque únicamente operativo (Solano, Bravo \& Giraldo, 2012) y asegurar el camino hacia el éxito competitivo y productivo. Los macroprocesos que componen cada uno de los factores presentan condiciones prácticas similares que los lleva a tener un mismo foco estratégico. De esta manera, las pequeñas organizaciones pueden enfocarse por mejorar las operaciones de cada uno de los factores propuestos, que finalmente afectan el desempeño de otros procesos con los que se tiene alta relación al compartir factor y esto, por último, puede mejorar el desempeño logístico y total de la organización.

Los futuros trabajos apuntan a expandir el análisis a otras regiones o países, la implementación del modelo usado en grandes empresas para comparar resultados y, finalmente, la implementación en empresas no solamente manufactureras, lo que implicará una adaptación del modelo a industrias de servicios y otros sectores. Asimismo, realizar un muestreo de manera totalmente aleatoria en una población determinada de empresas.

\section{Agradecimientos}

Al profesor Juan G. Arrieta-Posada del Departamento de Ingeniería de Producción de la Universidad EAFIT por su compromiso y dedicación durante el desarrollo de la investigación.

\section{Financiación}

Los autores recibieron apoyo financiero para la investigación, la autoría y la publicación de este artículo del Departamento de Descubrimiento y Creación de la Universidad EAFIT en Medellín, Colombia.

\section{Referencias}

Barbosa, D.H., \& Musetti, M.A. (2012). A survey on SMEs logistics performance in the capital goods industry: a comparative analysis. Production, 22(2), 249-258. https://doi.org/10.1590/s010365132012005000016

Battista, C., \& Schiraldi, M.M. (2013). The logistic maturity model: Application to a fashion company. International Journal of Engineering Business Management, 5(SPL.ISSUE). https://doi.org/10.5772/56838

Comrey, A.L., \& Lee, H.B. (2013). A First Course in Factor Analysis. In The Statistician (2 ${ }^{\text {nd }}$ Ed., Vol. 43, Issue 2). Psychology Press. https://doi.org/10.4324/9781315827506

Cronemyr, P., \& Danielsson, M. (2015). Process Management 1-2-3 - a maturity model and diagnostics tool. Total Quality Management \& Business Excellence, 24(April 2015), 37-41. https://doi.org/10.1080/14783363.2013.791114

Croxton, K.L., García-Dastugue, S.J., Lambert, D.M., \& Rogers, D.S. (2001). The Supply Chain Management Processes. The International Journal of Logistics Management, 12(2), 13-36. 
https://doi.org/10.1108/09574090110806271

Dziuban, C. D., \& Shirkey, E. C. (1974). When is a correlation matrix appropriate for factor analysis? Some decision rules. Psychological Bulletin, 81(6), 358-361. https://doi.org/10.1037/h0036316

Gelinas, R., \& Bigras, Y. (2004). The Characteristics and Features of SMEs: Favorable or Unfavorable to Logistics Integration? Journal of Small Business Management, 42(3), 263-278. https://doi.org/10.1111/j.1540-627x.2004.00111.x

Gorman, B.S., \& Primavera, L.H. (1983). The complementary use of cluster and factor analysis methods. Journal of Experimental Education, 51(4), 165-168. https://doi.org/10.1080/00220973.1983.11011856

Granadillo, H., Acosta, E.O., \& Mart, D. (2018). Diseño de un modelo de factores clave de capacidad logística para las pequeñas y medianas empresas del sector de confecciones de Cartagena, Colombia. Interciencia, 43(3), 215-218. https://www.redalyc.org/articulo.oa?id=339/33957185011

Hofmann, E., \& Rüsch, M. (2017). Industry 4.0 and the current status as well as future prospects on logistics. Computers in Industry, 89, 23-34. https://doi.org/10.1016/j.compind.2017.04.002

Huang, S.J., \& Han, W.M. (2006). Selection priority of process areas based on CMMI continuous representation. Information and Management, 43(3), 297-307. https://doi.org/10.1016/j.im.2005.08.003

Jovanović, I., Arsić, M., \& Nikolić, Đ. (2018). Entrepreneurial personality traits and SMEs profitability in transition economy. Serbian Journal of Management, 13(1), 89-104. https://doi.org/10.5937/sjm13-13087

Keh, H.T., Nguyen, T.T.M., \& Ng, H.P. (2007). The effects of entrepreneurial orientation and marketing information on the performance of SMEs. Journal of Business Venturing, 22(4), 592-611. https://doi.org/10.1016/j.jbusvent.2006.05.003

Kitsios, F., Kamariotou, M., Madas, M.A., Fouskas, K., \& Manthou, V. (2019). Information systems strategy in SMEs: critical factors of strategic planning in logistics. Kybernetes, 49(4), 1197-1212. https://doi.org/10.1108/K-10-2018-0546

Laufs, K., Bembom, M., \& Schwens, C. (2016). CEO characteristics and SME foreign market entry mode choice: The moderating effect of firm's geographic experience and host-country political risk. International Marketing Review, 33(2), 246-275. https://doi.org/10.1108/IMR-08-2014-0288

Lloret-Segura, S., Ferreres-Traver, A., Hernández-Baeza, A., \& Tomás-Marco, I. (2014). El análisis factorial exploratorio de los ítems: Una guía práctica, revisada y actualizada. Anales de Psicologia, 30(3), 1151-1169. https://doi.org/10.6018/analesps.30.3.199361

Nyoni, T., \& Bonga, W.G. (2018). Anatomy of the Small \& Medium Enterprises (SMEs) Critical Success Factors Anatomy of the Small \& Medium Enterprises (SMEs) Critical Success Factors (CSFs) in Zimbabwe : Introducing the 3E Model. Journal of Business and Management (DRJ-JBM, 1(2), 01-18. https://ssrn.com/abstract $=3130094$

Olivos, P.C., Carrasco, F.O., Flores, J.L.M., Moreno, Y.M., \& Nava, G.L. (2015). Modelo de gestión logística para pequeñas y medianas empresas en México. Contaduria y Administracion, 60(1), 181203. https://doi.org/10.1016/S0186-1042(15)72151-0

Osborne, J.W., Costello, A.B., \& Kellow, J.T. (2011). Best Practices in Exploratory Factor Analysis. In Best Practices in Quantitative Methods (Issue September). 
https://doi.org/10.4135/9781412995627.d8

Pennypacker, J.S., \& Grant, K.P. (2003). Project Management Maturity: An Industry Benchmark. Project Management Journal, 34(1), 4-11. https://doi.org/10.1177/875697280303400102

Puertas, R., Martí, L., \& García, L. (2014). Logistics performance and export competitiveness: European experience. Empirica, 41(3), 467-480. https://doi.org/10.1007/s10663-013-9241-z

Revelle, W., \& Zinbarg, R. (2009). Coefficientes Alpha, Beta, Omega and the GLB. Psychometrika, 74(1), 145-154.

Sledgianowski, D., Luftman, J.N., \& Reilly, R.R. (2011). Development and Validation of an Instrument to Measure Maturity of IT Business Strategic Alignment Mechanisms. Information Resources Management Journal, 19(3), 18-33. https://doi.org/10.4018/irmj.2006070102

Solano, M.A., Bravo, J.J., \& Giraldo, J.A. (2012). Metodología de mejoramiento en el desempeño de sistemas de producción. Aplicaciones en Pymes de la confección. Ingeniería y Competitividad, 14(2), 37-52. https://doi.org/10.25100/iyc.v14i2.2653

Song, G., Song, S., \& Sun, L. (2019). Supply chain integration in omni-channel retailing: a logistics perspective. International Journal of Logistics Management, 30(2), 527-548. https://doi.org/10.1108/IJLM-12-2017-0349

Tracey, M. (1998). The Importance of Logistics Efficiency to Customer Service and Firm Performance. The International Journal of Logistics Management, 9(2), 65-81. https://doi.org/10.1108/09574099810805843

Tran, L. (2019). The factors affecting Logistics Service Quality - case study at Saigon New Port Logistics (SNPL), Vietnam. https://www.theseus.fi/handle/10024/166324

Valadares de Oliveira, M.P., Bronzo Ladeira, M., \& McCormack, K.P. (2011). The Supply Chain Process Management Maturity Model - SCPM3. Supply Chain Management - Pathways for Research and Practice. https://doi.org/10.5772/18961

Williams, B., Onsman, A., \& Brown, T. (2010). Exploratory factor analysis: A five-step guide for novices. Australasian Journal of Paramedicine, 8(3), 1-13. https://doi.org/10.33151/ajp.8.3.93

Wynn, M.G., Turner, P., \& Lau, E. (2013). E-business and process change: Two case studies (towards an assessment framework). Journal of Small Business and Enterprise Development, 20(4), 913-933. https://doi.org/10.1108/JSBED-03-2012-0044

Yang, B., \& Lu, J. (2017). Empirical Research on the Factors Affecting Core Competence Evolution of Logistics Enterprises Based on Service Innovation. Proceedings of the 2017 International Conference on Humanities Science, Management and Education Technology (HSMET 2017), 96(Hsmet), 1205-1209. https://doi.org/10.2991/hsmet-17.2017.222

Zimon, D., Madzik, P., \& Sroufe, R. (2019). Management systems and improving supply chain processes: Perspectives of focal companies and logistics service providers. International Journal of Retail and Distribution Management. https://doi.org/10.1108/IJRDM-04-2019-0107 